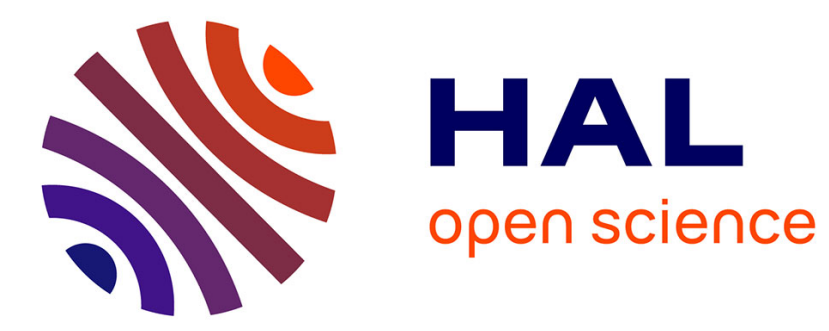

\title{
Quasi-elastic neutron scattering study of a re-entrant side-chain liquid-crystal polyacrylate
}

Ludovic Benguigui, Laurence Noirez, R. Kahn, P. Keller, M. Lambert, E.

Cohen de Lara

\section{- To cite this version:}

Ludovic Benguigui, Laurence Noirez, R. Kahn, P. Keller, M. Lambert, et al.. Quasi-elastic neutron scattering study of a re-entrant side-chain liquid-crystal polyacrylate. Journal de Physique II, 1991, 1 (4), pp.451-458. 10.1051/jp2:1991179 . jpa-00247529

\section{HAL Id: jpa-00247529 https://hal.science/jpa-00247529}

Submitted on 1 Jan 1991

HAL is a multi-disciplinary open access archive for the deposit and dissemination of scientific research documents, whether they are published or not. The documents may come from teaching and research institutions in France or abroad, or from public or private research centers.
L'archive ouverte pluridisciplinaire HAL, est destinée au dépôt et à la diffusion de documents scientifiques de niveau recherche, publiés ou non, émanant des établissements d'enseignement et de recherche français ou étrangers, des laboratoires publics ou privés. 
Classification

Physics Abstracts

$61.12-61.30$

\title{
Quasi-elastic neutron scattering study of a re-entrant side-chain liquid-crystal polyacrylate
}

\author{
L. Benguigui ( $\left.{ }^{1, *}\right)$, L. Noirez, R. Kahn, P. Keller, M. Lambert, E. Cohen de Lara ( ${ }^{2}$ ) \\ (1) Laboratoire LEon Brıllouin (CEA-CNRS), CEN Saclay 91191 Gif-sur-Yvette, France \\ ( $\left.{ }^{2}\right) \operatorname{DRP}\left({ }^{* *}\right)$, Univ. Pierre et Marie Curıe 75252 Paris Cedex 05, France
}

(Received 26 October 1990, revised 14 January, accepted 15 January 1991)

\begin{abstract}
Résumé. - Nous présentons une premıère êtude dynamique par diffusion quası-élastique des neutrons, d'un échantillon de polyacrylate mésomorphe en peigne dans chacune des phases : isotrope, nématique, smectıque et nématıque rentrante. On montre que le mouvement et/ou la mobılité des protons du mésogène se restreınt à mesure que la température dimınue après la transition isotrope-nématique. Contrairement à la transition $\mathrm{N}-\mathrm{S}_{\mathrm{A}}$, les transitions $\mathrm{I}-\mathrm{N}$ et $\mathrm{S}_{\mathrm{A}}-\mathrm{N}_{\mathrm{Re}}$ correspondent a une discontinuité dans la courbe du Facteur de Structure Incohérent Elastique (rapport: intensité élastıque/intensıté totale) en fonctıon de la température; l'ordre local semble donc très proche pour les phases nématıque et smectıque.
\end{abstract}

\begin{abstract}
We present a first investigation of the dynamics of a side chain liquid crystal polyacrylate in the isotropic (I), nematic $(N)$, smectic $A\left(S_{A}\right)$, and re-entrant nematic $\left(N_{R e}\right)$ phases by means of quasi-elastic neutron scattering. The motion or/and the mobılity of the mesogen protons decreases as soon as the temperature decreases after the isorropic-nematic transition. The I-N and $\mathrm{S}_{\mathrm{A}}-\mathrm{N}_{\mathrm{Re}}$ transitions correspond to a jump in the curve of the Elastic Incoherent Structure Factor (ratio: elastic scattenng/total scattering) versus temperature, on the other hand the transition $\mathrm{N}-\mathrm{S}_{\mathrm{A}}$ occurs without any change of slope. We conclude that the local order is very similar in the nematic and the smectic $A$ phases.
\end{abstract}

\section{Introduction.}

The re-entrant phenomenon is relatively frequent in liquid crystals [1] and it was also recently observed in the case of polymer liquid crystals [2]. The latter is constitued by mesogenic units linked to the backbone of a linear polymer via a small aliphatic chain called spacer (comb-like liquid crystal polymers). The polymer we studied is the one described in reference [2b]; it presents

(*) Solıd State Institute Technion, 32000 Haifa,Israel

$\left({ }^{* *}\right)$ associé au CNRS. 
a re-entrant nematic phase and corresponds to the following formula for the labelled species :

$$
\begin{aligned}
& {\left[\mathrm{CD}_{2}-\mathrm{CD}\right]_{n}-} \\
& \quad \mathrm{CO}_{2}-\left(\mathrm{CH}_{2}\right)_{6}-\mathrm{O}-\varphi-\varphi-\mathrm{CN}
\end{aligned}
$$

where $\mathrm{D}$ designates the deuterium atom.

This deuterated polymer has a molecular weight of 130000 and a polydispersity of 2.7, it presents the following sequence of phases [2] with decreasing temperature:

Isotropic (I) $-122^{\circ} \mathrm{C}$ Nematic $(N)-112^{\circ} \mathrm{C}$ Smectic $A\left(S_{A}\right)-83^{\circ} \mathrm{C}$ Reentrant Nematic $\left(\mathrm{N}_{\mathrm{Re}}\right)$ $35^{\circ} \mathrm{C}$ Glassy

This side-chain polymer is particularly interesting because the backbone takes different shapes in each of the two nematic phases. In the $N$ phase (above the $S_{A}$ phase) the polymer backbone is mostly perpendicular to the mean direction of the mesogens; the global shape of the polymer is an oblate ellipsoid, as well as in the $S_{A}$ phase itself. While in the $N_{R e}$ phase the polymer backbone is more parallel to the mesogen direction; the global shape is a prolate ellipsoid[2.b].For the isotropic phase the backbone has a spherical symmetry as expected in such a phase. The change in the shape of backbone in the two nematic phases is very surprising and is not yet clearly understood. Notice that this polymer has also also studied by dielectric relaxation [2c] and compared to its low molecular weight homologous.

In order to study more fully the characteristics of the different phases, we undertook a molecular dynamic study by means of incoherent quasi-elastic neutron scattering. If there is such a dramatic change of the backbone conformation when passing from the $S_{A}$ phase to the $N_{R e}$ phase, we expect the local molecular dynamics to present also some difference between the two phases.

This paper is organized as follows : section 2 is devoted to a commented presentation of the general results obtained with this liquid crystal polymer. Section 3 begins with an analysis of the different motions which are possible in the liquid crystal polymer structure. The results expressed in terms of EISF are then discussed . Different physical situations are proposed and the conclusions are drawn in section 4.

\section{Experiments and Results.}

In our experiments we used a sample containing the polymer with the deuterated backbone in bulk. Since the scattering cross-section of the deuterium atom is very small compared to that of the hydrogen, we measure only the contribution of the hydrogen atoms to the scattering intensity and so investigate the motions of the protons of the mesogens.

The experiments were performed on the time of flight spectrometer (MIBEMOL) at the Laboratoire Léon Brillouin. The wavelength used was fixed equal to $10 \AA$ The spectra were recorded for four scattering angles corresponding to $0.44,0.60,0.75$ and $1.16 \AA^{-1}$ for the value of the elastic momentum transfer. The sample (a disk-like cell of $1 \mathrm{~mm}$ thickness, $15 \mathrm{~mm}$ diameter) was positioned in a temperature controlled furnace, cooled from the isotropic phase without any process of alignment of the mesogenic units. So our sample can be seen as a powder sample. When the faces of the sample are perpendicular to the incoming neutron beam, the fraction of transmitted beam is about 50 per cent. This low value shows that we must take care in our planar geometry of the phenomenon of the multiple scattering which becomes of importance from neutrons which have been scattered twice by 90 degrees. This multiple scattering is known to produce two effects:

1) A non-zero quasi-elastic scattering when $Q$ becomes very small: When the Elastic Incoherent Scattering Factor EISF (defined by the ratio of the elastic intensity over the total intensity) in the direction $\pi / 2$ is not too far from 1 ( 0.8 in our case) the quasi-elastic component of the double scattering at very small angle has essentially the energy width of the scattering function at $\theta=\pi / 2$. 
2) A systematic decrease of the elastic scattering. This effect is maximum in the low $Q$ region and decreases when $\theta$ increases. The influence of the multiple scattering on the EISF has been quantitatively calculated in the quasi-elastic limit [3]. The error is maximum (20\%) in the low $Q$ region and decreases to $10 \%$ at the highest $Q$ value used $\left(1.16 \AA^{-1}\right)$. At this $Q$ value, the statistical accuracy is of the same order of magnitude (10\%). So, the second effect will not be taken into account as far as we build the analysis on the data obtained only at wide angles $\left(Q=1.16 \AA^{-1}\right)$. Standard procedures were used to determine the instrumental resolution (35 $\mu \mathrm{ev}$ FWHM). A typical spectrum is represented figure $1:$ the spectra were analysed as the superposition of an elastic and a quasi-elastic scattering. This latter was modelized by a simple Lorentzian curve.

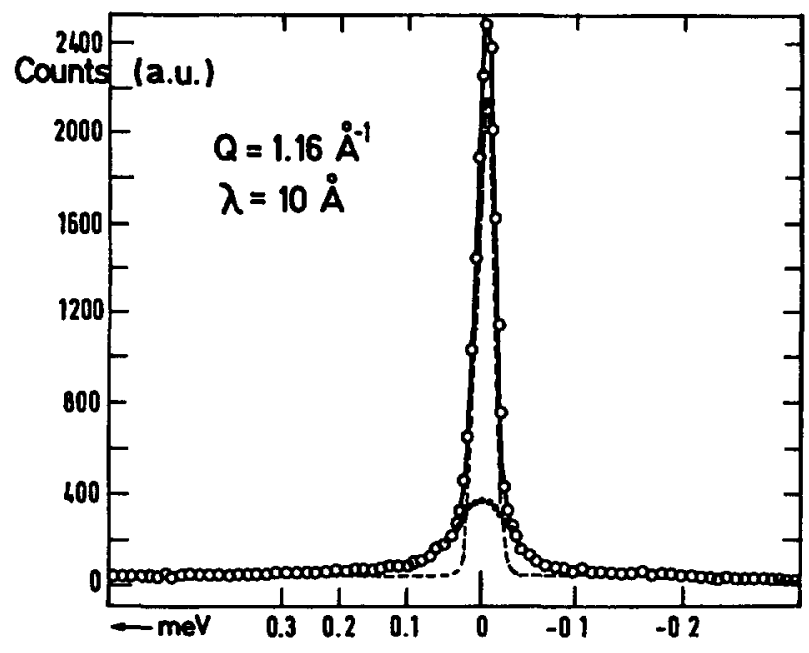

Fig. 1. - Time of fight spectrum for the side-chain polyacrylate deuterated on the backbone at $138^{\circ} \mathrm{C}$

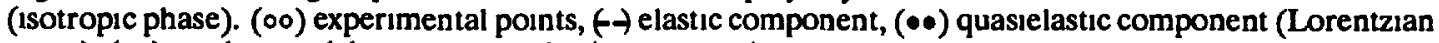
curve), (-) total curve (elastıc + quasielastic scatterıng).

The general results were that the quasi-elastic scattering integrated intensity $I_{\text {qe }}$ decreases when the temperature decreases, while the elastic scattering intensity $I_{e}$ increases. In figures 2 and 3 , we present the variation of $I_{\mathrm{qe}}$ and $I_{\mathrm{e}}$ versus temperature $T$ for $Q=116 \AA^{-1}$. We distinguish clearly three domains : the isotropic phase, the high temperature nematic and the smectic $A$ phases, and the reentrant nematic phase. This distinction coincides with the three different backbone conformations : spherical in the isotropic phase, oblate in the $\mathrm{N}$ and $\mathrm{S}_{\mathrm{A}}$ phases and prolate in the $\mathrm{N}_{\mathrm{Re}}$ phase. We note also discontinuities of $I_{\mathrm{qe}}$ at the I-N transition and at the $S_{\mathrm{A}}-\mathrm{N}_{\mathrm{Re}}$ transition. This seems to indicate that the I-N and the $S_{A}-N_{R e}$ are first order transitions.

In figure 2 the variation of the Lorentzian half width $\Gamma$ is also given as a function of $T$ : contrary to $I_{e}(T)$ and $I_{\mathrm{qe}}(T)$, the curve $\Gamma(T)$ does not exhibit clearly discontinuities at the $I-N$ and at the $\mathrm{S}_{\mathrm{A}}-\mathrm{N}_{\mathrm{Re}}$ transitions. $\Gamma$ seems constant in the isotropic phase, and decreases slightly with $\mathrm{T}$ from the nematic phase. The values of $\Gamma$ are in a ratio of $1.5-2$ between the two nematic phases. The characteristic time $\tau \sim 1 / \Gamma$ appears to increase in the liquid crystalline phases by decreasing the temperature; the mesomorphic phases accelerate the immobilizing process of the protons when the temperature decreases. 


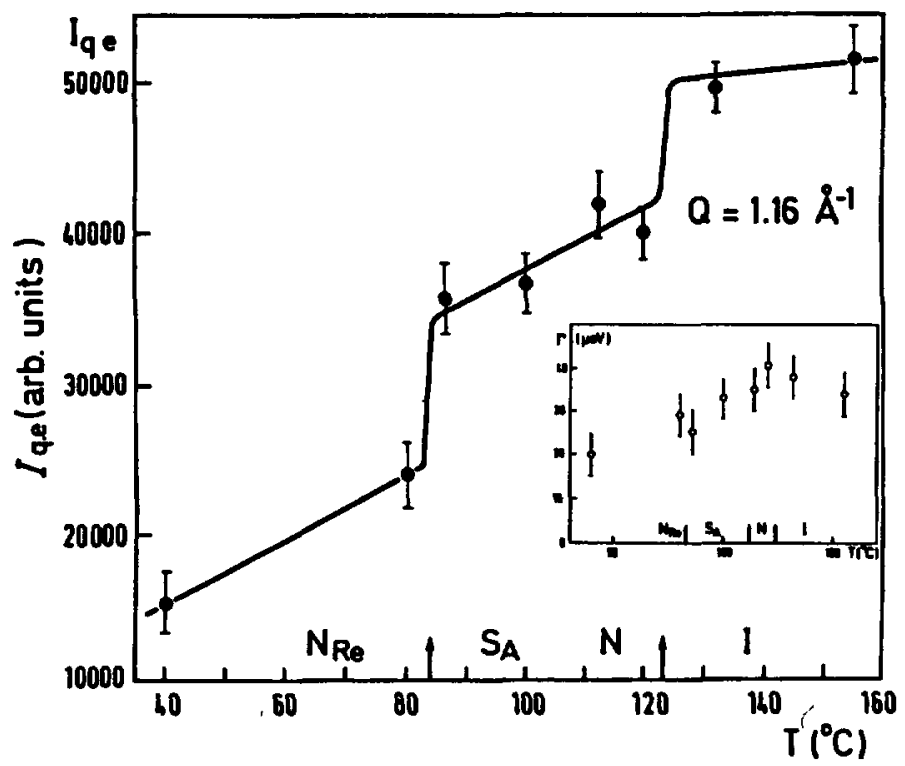

Fig. 2. - The quasi-elastic scattering intensity $I_{q e}$ versus $T$, in the different phases for a value of the momentum transfer $Q=116 \AA^{-1}$. Included in this figure, the vanation of the half width at half maximum of the Lorentzian $\Gamma$ of the quasi-elastic scattering, with $T$.

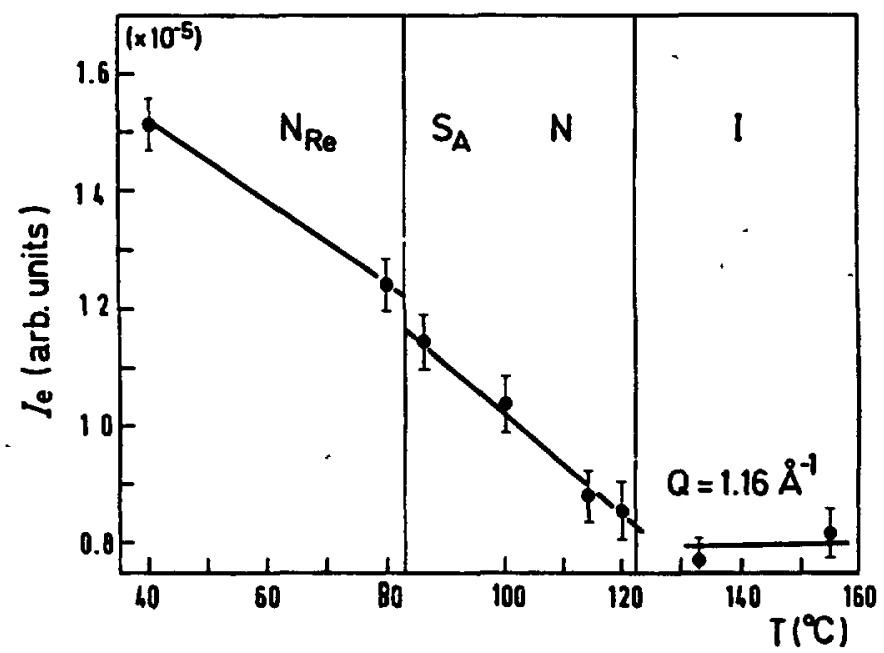

Fig. 3. - The elastic scattering intensity $I_{e}$ versus $\mathrm{T}$ for a momentum transfer $Q=1.16 \AA^{-1}$. Note, as in figure 2 discontınutties at the isotropıc-nematic and the smectıc A-reentrant nematıc phases.

In order to give a measure of the volume involved in the motion of the mesogen protons, we calculated the EISF and compare this quantity to that obtained with different motion models for which we determine a characteristic length "a" related to the largest trajectory swept by the protons. 
The EISF noted $E(Q a)$ is equal to $\frac{I_{\mathrm{e}}}{I_{\mathrm{e}}+I_{\mathrm{qe}}}$. It is observed that the quasi-elastic scattering $I_{\mathrm{qe}}$ is dependent of $Q$ as $I_{\mathrm{qe}}=A+B Q^{2}$. It is very likely that the term $\mathrm{A}$ is an effect of the multiple scattering. So, for a first analysis essentially qualitative we can take the multiple scattering effect into account simply by replacing $I_{\mathbf{q e}}$ by $I_{\mathrm{qe}}-A$ in the expression of the EISF. This gives a correction of about $10 \%$. In figure 4, we present the EISF as a function of $T$ for $Q=1.16 \AA$ (the variation of the EISF versus $Q$ without corrections of multiple scattering effect is also given at the top of the figure 4).

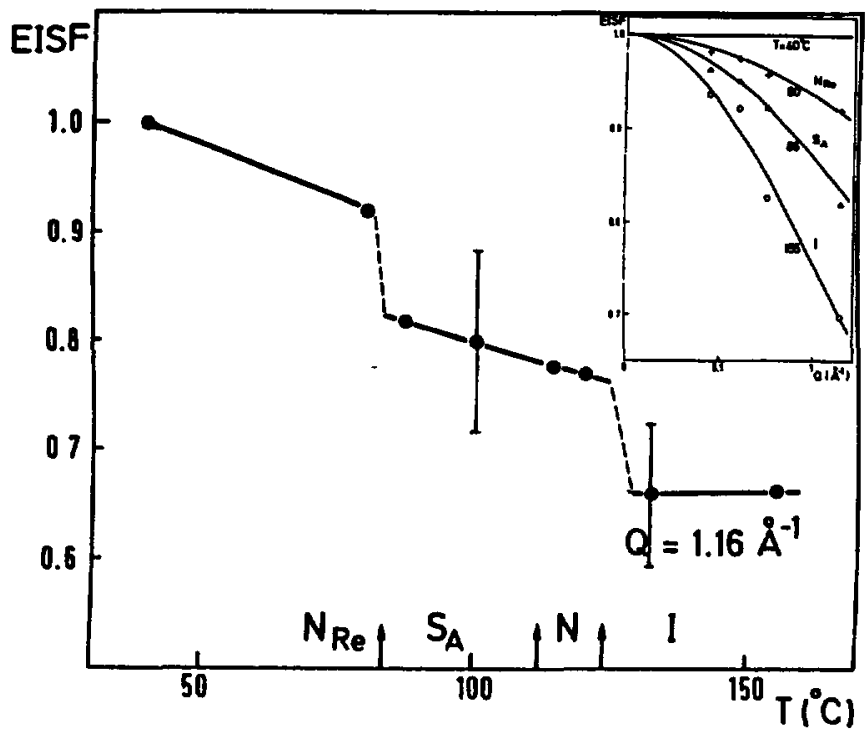

Fig. 4. - EISF as a function of the temperature $\left({ }^{\circ} \mathrm{C}\right)$ for $Q=1.16 \AA^{-1}$. Included at the top of the figure, the EISF versus $Q$, at different temperatures.

\section{Discussion.}

The increase of the EISF when $T$ decreases (Fig. 4) can be explained following two possibilities. Either there is a decrease of the number of protons contributing to the quasi-elastic scattering, because the characteristic time of their motion becomes too small [4]. Or there is a decrease of the volume swept by the protons. Obviously, these two possibilities can occur together. In the compound studied there are two kinds of protons : the twelve protons of the spacer (the $\mathrm{C}_{6} \mathrm{H}_{12}$ chain between the backbone and the liquid crystal part) and the eight of the benzene rings. Because of the direct bond between the two rings, the benzene protons should move together in a motion which is the superposition of a rotation around the symmetry axis of the rings and of the motion of this axis. In principle, the motion of the spacer protons should be more complicated if the chain can have numerous conformations. In fact, the spacer is linked to the backbone and to the benzene rings and, as shown by X-ray [5], the terminal chains of a liquid crystal are in an elongated configuration. Thus the motion of the protons of the spacer takes place with these two constraints : an elongated chain and a chain linked to the backbone and to the benzene rings. 
Starting with assumption, we propose to interprete the value of the EISF at first in the isotropic state.

The EISF, noted $E(Q a)$, is analysed in the isotropic phase using three different models according to the type of motion assumed. The results of this analysis is not very model dependent. Indeed, because the $Q$-range chosen is short, the EISF depends very little on the model as long as $Q . a \lesssim 1$. The three possibilities studied are the following (see Ref.[6] for the corresponding formulas).

The values of "a" are obtained by comparing the experimental EISF to that calculated with $Q=1.16 \AA^{-1}$.

a) The protons describe a circular motion of radius $a$. We have [6] (for a powder sample):

$$
E(Q a)=\frac{1}{N} \sum_{k=1}^{N} j_{\circ}\left(2 Q a \sin \frac{k \pi}{N}\right)
$$

where $j_{o}(x)=\frac{\sin x}{x}, N$ is the number of the positions on the circle from which the protons jumps from one to another. We took $N=5$, since for $N \gtrsim 5$, we almost recover a continuous diffusive motion.

b) The protons undertake a diffusive motion on a sphere of radius $a$ and the EISF is :

$$
E=\left[j_{0}(Q a)\right]^{2}
$$

c) The protons undertake a diffusive motion inside a hard sphere of radius $a$ :

$$
E=\left[\frac{3 j_{1}(Q a)}{Q a}\right]^{2}
$$

where $j_{1}(x)$ is the first spherical Bessel function.

In a first approach one assumes that the biphenyl group describes a circular motion of radius $a_{b} . a_{b}$ is of the same size as the radius of the benzene rings, $a_{b} \simeq 2.15 \AA$ [7]. We can write now the EISF as the sum of the EISF of the spacer protons and of that of the biphenyl :

$$
E=\frac{8}{20} E_{\mathrm{b}}\left(Q a_{\mathrm{b}}\right)+\frac{12}{20} E_{\mathrm{s}}\left(Q a_{\mathrm{s}}\right)
$$

where $a_{s}$ is the characteristic length of the spacer. Whatever the model (circular motion, motion on or inside a sphere of radius $a_{\mathrm{s}}$ ), the value $a_{\mathrm{b}} \simeq 2.15 \AA$ does not allow to obtain a value of $a_{\mathrm{s}}$. Even with $a_{s} \equiv 0$ the computed value of the EISF is much lower than the experimental one and corresponds to $a_{b}=1.75 \AA$. Since the values of $a_{b}$ are smaller than the radius of the benzene rings ( $2.15 \AA$ ), we conclude that the protons of the rings do not sweep a complete rotation around the long axis. However, we cannot exclude libration motions of relatively small amplitude. It is in marked contrast with the small molecule liquid crystals [8]. Indeed in the case of TBBA [9], the protons of the core rotate and contribute to the quasi-elastic scattering in all its liquid crystal phases (for a similar window of characteristic times). This is in accordance with what we know about the dynamic properties of the polymer liquid crystals for which NMR experiments have been done [10]: the characteristic times for the $180^{\circ}$-phenyl ring jump are much larger by several orders of magnitude [2c], [10]:

-Thus, if we now take the mesogen as a whole and determines its characteristic length " $a$ ". We obtain for the isotropic phase $a=0.8-1.4 \AA$ and a characteristic time of $\tau=19 \times 10^{-11}$ sec. 
The conclusion we adopted namely that the protons of the mesogens do not rotate freely around the long axis for this time of scale is clearly also valid in the liquid crystal phases.

At the isotropic-nematic transition there is an upward jump in the EISF curve versus temperature, particularly well observed at $Q=1.16 \AA$ (see Fig.4). We explain this increase of the EISF by a decrease of $a$, which does not affect the number of the protons involved. This last point is deduced from the small variation, if any of $\Gamma$ (included in Fig.2). Indeed, if there were a slowing down of the proton motion we would also observe a steep decrease in the $\Gamma$ curve. Since it is not the case, we conclude that the increase of the EISF at the I-N transition is mostly due to a decrease of the characteristic length " $a$ ". Notice that $\Gamma$ appears constant in the isotropic phase and only begins to decrease at the I-N transition without any apparent jump at the $N-S_{A}$ transition : at this local scale, the dynamics of the interactions is thus similar in the $N$ and $S_{A}$ phases. In the same way, the upward jump of the EISF observed at the $S_{A}-N_{R e}$ transition could be interpreted by a decrease of the characteristic length " $a$ " which implies that the reentrant nematic phase is controlled by other interactions.

\section{Conclusion.}

We presented the first study of the dynamics of a comb-like liquid crystal polymer, by means of quasi-elastic neutron scattering. One important result is that the high temperature nematic phase and the smectic phase appear identical from a dynamical point of view (in our window of measurement) whereas the re-entrant nematic phase differs deeply. Indeed, if one describes the motion of the protons of the mesogen by a specific length $a$ and a time $\tau$, one observes that $\tau$ varies weakly with $T$, contrary to $a$. This length a has downward discontinuities at the isotropic-nematic transition and at the smectic A-re-entrant nematic transitions but not at nematic-smectic $A$ transition, confirming that in these last two phases the local dynamics and therefore, the interactions, are very similar.

Another interesting result is the value of the length $a=0.8-1.4 \AA$ which is too weak to correspond to a complete rotation of the whole mesogen around its long axis. This should be at variance with the case of small molecule liquid crystals for which the motion of complete rotation of the aromatic part is faster and can be measured by quasielastic scattering. From a physical point of view, the exact kind of motion of the protons of the spacer and of those of the benzene stays unknown (larger values of the momentum transfer $Q$ are needed). It is also obvious that a selective labelling of the spacer or the benzene protons could allow a simpler analysis of the motions. New experiments are planned in the near future with thinner aligned liquid crystal polymers in order to reduce the multiple scattering and to analyse the geometry of the motion in space.

\section{Acknowledgements.}

It a pleasure to thank J.P. Cotton for his constructive remarks and $G$. Coddens for enlightening discussions about the spectrometer MIBEMOL.

\section{References}

[1] a) CLADIS P.E., Phys. Rev. Lett. 35, (1979) 48.

b) Hardouin F., Levelut A.M., Achard M.A., Sigaud G., J. Chim. Phys. 1, (1983) 53. 
[2] a) Le baRny P., Dubois J.C., Friedrich C., NOEl C., Polymer Bull 15, (1986) 394.

b) NoIrez L., KEller P., DAvidson P., HARdouin F, CơTTON J.P., J. Phys. France 49, (1988) 1993.

c) PARNeIX J.P., NJEUMO R., LEgRAND C., LE BARNY P., DuBoIs J.C., Liquid Crystals 2, (1987) 167.

[3] SEARS V.F., Adu: Phys. 24, (1975) 1.

[4] From the resolution of the spectrometer, all hydrogene whose motion is associated to a characteristic time higher than $10^{-10} \mathrm{sec}$ will be seen as "at rest" and therefore will give a purely elastic scattering.

[5] DAVIDSON P., private communication.

[6] BEE M., Quasi-Elastic Neutron Scattering, Adam Hilger Ed. (1988)

[7] Volino F., DianouX A.J. and HeRvet H.,J. Phys. France Colloq. (1976) C3-37, C3-55.

[8] a) ChledowsKa K., Chrusciel D., JANIK J.A. , JANIK J.M., KREESE H., STETTIN H., OTNES K., STANEK T., URBAN, S. and Wrobel S., Liquid Cystals 3, (1988) 1339.

b) MONNERIE L., LAUPRETRE F., NOEL C., Liquid Cystals 3, (1988) 1.

[9] Dianoux AJ. and Volino F., J. Phys.France 40, (1979) 181.

[10] a) For the dielectric relaxation see BORTMUTH FJ. and HAASE W., MoL Cryst. Liq. Cryst. 153, (1987) 207 and ZENTEL R., STROBL, G. R., RINGSDORF, H., Macromolecules 18, (1985) 960.

b) For acoustic relaxation see BENGUIGUI L. and RON P., Mol Cryst. Liq. Cryst. 153, (1987) 241.

c) For spectroscopic techniques see, MONNERIE L., LAUPRETRE F. and NOEL C., Liquid Crystals 3, (1988) 1 and 1013; BOEFFEL C., SpIEss H.W., "Side Chain liquid crystal Polymers" C.B. Mc Ardle Ed. (1989) Chapter 8; and also PSCHORN U., SPIESS H.W., HISGEN B., RINGSDORF H., Makromol Chem. 187 (1986) 2711.

Cet artıcle a été ımprımé avec le Macro Package "Edıtıons de Physique Avril 1990". 